\title{
Clinical predictors of antipsychotic use in children and adolescents with autism spectrum disorders: a historical open cohort study using electronic health records
}

\author{
Johnny Downs ${ }^{1} \cdot$ Matthew Hotopf $^{1} \cdot$ Tamsin Ford $^{1,3} \cdot$ Emily Simonoff $^{1}$ • \\ Richard G. Jackson $^{1,2} \cdot$ Hitesh Shetty $^{2} \cdot$ Robert Stewart $^{1,2} \cdot$ Richard D. Hayes $^{1}$
}

Received: 1 May 2015 / Accepted: 28 September 2015 / Published online: 15 October 2015

(C) The Author(s) 2015. This article is published with open access at Springerlink.com

\begin{abstract}
Children with autism spectrum disorders (ASD) are more likely to receive antipsychotics than any other psychopharmacological medication, yet the psychiatric disorders and symptoms associated with treatment are unclear. We aimed to determine the predictors of antipsychotic use in children with ASD receiving psychiatric care. The sample consisted of 3482 children aged 3-17 with an ICD-10 diagnosis of ASD referred to mental health services between 2008 and 2013. Antipsychotic use outcome, comorbid diagnoses, and other clinical covariates, including challenging behaviours were extracted from anonymised patient records. Of the 3482 children (79\% male) with ASD, $348(10 \%)$ received antipsychotic medication. The fully adjusted model indicated that comorbid diagnoses including hyperkinetic (OR 1.44, $95 \%$ CI 1.012.06), psychotic $(5.71,3.3-10.6)$, depressive $(2.36,1.37-$ 4.09), obsessive-compulsive $(2.31,1.16-4.61)$ and tic disorders $(2.76,1.09-6.95)$ were associated with antipsychotic use. In addition, clinician-rated levels of aggression, selfinjurious behaviours, reduced adaptive function, and overall parental concern for their child's presenting symptoms were significant risk factors for later antipsychotic use. In
\end{abstract}

Electronic supplementary material The online version of this article (doi:10.1007/s00787-015-0780-7) contains supplementary material, which is available to authorized users.

Johnny Downs

johnny.downs@kcl.ac.uk

1 Institute of Psychiatry, Psychology and Neuroscience, King's College London, De Crespigny Park, Box 63, SE5 8AF London, UK

2 South London and Maudsley NHS Foundation Trust, London, UK

3 University of Exeter Medical School, Exeter, UK
ASD, a number of comorbid psychiatric disorders are independent predictors for antipsychotic treatment, even after adjustment for familial, socio-demographic and individual factors. As current trial evidence excludes children with comorbidity, more pragmatic randomised controlled trials with long-term drug monitoring are needed.

Keywords Child and adolescence $\cdot$ Autism spectrum disorders $\cdot$ Antipsychotic medications $\cdot$ Psychiatric comorbidity $\cdot$ Challenging behaviours

\section{Introduction}

Antipsychotics are the most common psychotropic medication prescribed to children with autism spectrum disorders (ASD) [1]. US-based studies suggest between 20 and $34 \%$ of children with ASD receive antipsychotics [2, 3]. Rates are lower in Europe, between 7 and $11 \%$ [4, 5], but appear to be increasing [6]. Two atypical antipsychotics in particular are most commonly used, risperidone and aripiprazole, which have been demonstrated to be effective in reducing "irritability" in children with ASD, but show limited impact on the core features of ASD [7].

Clinicians and families face a difficult task when deciding whether antipsychotic treatment is indicated. Evidence from antipsychotic trials in childhood ASD is derived from samples that bear little resemblance to children typically seen in clinical practice, as they exclude children with formally diagnosed psychiatric comorbidity [8]. Another problem is the almost exclusive focus of trials on irritability as a target symptom in ASD. Irritability is a highly prevalent symptom in clinical settings, it has no standard taxonomy, and is associated with most childhood mental health problems [9]. Therefore, based on trial evidence, 
the type and severity of childhood ASD-related irritability symptoms, which warrant antipsychotic treatment, are unclear. Furthermore, antipsychotic medication does not have UK marketing authorisation for use in childhood ASD, although risperidone is licensed for use in the shortterm management of aggression in children with conduct disorder [10]. Balancing antipsychotic risk-benefit profiles is further complicated by little safety evidence being available for children with ASD [11, 12]. Antipsychotic use for children in general is associated with a number of adverse health outcomes, most commonly extrapyramidal side effects, obesity and hyperprolactinaemia [13]. Given the limited evidence base, NICE guidelines advocate cautious antipsychotic prescribing in children with ASD and only to treat severe challenging behaviours (also known as 'behaviours that challenge') such as aggression, self-injury and impulsive/dangerous behaviours [14].

It remains unclear how current evidence, licensing and guidance for antipsychotic use in children with ASD are applied clinically [15]. There are very few UK-based naturalistic studies of prescribing in children with $\mathrm{ASD}$, and, as yet, no examinations of the diagnostic predictors of antipsychotic use [4]. Comorbid psychiatric disorders are common (and frequently multiple) in children with autism spectrum disorders and may be targets for intervention [16]. Current knowledge is largely based on parent reports in US surveys which indicate that antipsychotics are used predominantly to treat comorbid diagnoses (e.g. depression, bipolar, anxiety, conduct disorder and attention deficit hyperactivity disorders) in children with ASD [2, 3]. However, these findings may not generalise to non-US clinical populations as US antipsychotic marketing [17], prescribing policy [18, 19] and practice differ markedly to the other Western Countries $[1,20]$. Given that the majority of the aforementioned studies report cross-sectional findings from retrospective parental accounts of both comorbidity and past medication use, the direction of effect is unclear, and recall bias may obscure true prescribing patterns. Furthermore, these studies do not account for important confounding factors, such as psychosis, adaptive function, and intellectual disability that may lead to an overestimate of the association between certain comorbidities and antipsychotic use.

To clarify how antipsychotics are used in childhood ASD, we explored the clinical factors that predicted antipsychotic prescribing. We conducted a historical cohort study using the anonymised electronic health records of children with ASD treated by UK child and adolescent mental health services (CAMHS). As challenging behaviours (or 'behaviours that challenge') are symptoms that cut across most childhood psychopathology, we hypothesised that the common psychopathologies are comorbid with ASD including hyperkinetic, oppositional and conduct, depression and anxiety disorders would all show longitudinal associations with antipsychotic use. We also examined whether associations between comorbidity and antipsychotics were attenuated after we controlled for challenging behaviours, given that these are the most common non-psychotic symptoms formally recognised as targets for antipsychotic treatment by current national ASD management guidelines [14].

\section{Methods}

\section{Study setting}

This study used data extracted from the anonymised, electronic clinical records of children referred to South London and Maudsley NHS Foundation Trust (SLaM) between 1 January 2008 and 31 December 2013. Over this period, SLaM provided all aspects of specialist mental healthcare to a catchment population of approximately 300,000 children resident within four London boroughs (Lambeth, Southwark, Lewisham, Croydon). In addition to the district services, SLaM provided specialist inpatient and outpatient ASD assessment and treatment services for young people from across the UK. Each borough had a dedicated multidisciplinary service for children, which accepted referrals for school age children (4-18 years; exceptionally cases are accepted below this age) with suspected or previously confirmed ASD, displaying emotional or behavioural difficulties. Children were referred from primary care, child health, and educational and social care services, and typically underwent a multidisciplinary assessment by CAMHS clinicians. Primary and secondary psychiatric disorders were diagnosed by CAMHS using the ICD10 multi-axial classification system [21]. Semi-structured validated assessments, for example, the Autism Diagnostic Observation Schedule (ADOS) [22] were used if an ASD diagnosis was unclear after initial assessment (the SLaM autism pathway is available from the author on request). Compared with expert consensus, there is a high specificity for ASD diagnoses by clinicians working at a district level [23]. Socio-demographic characteristics and clinical information were recorded using computerised assessment proforma, which included the Strengths and Difficulties Questionnaire (SDQ) [24].

The Clinical Record Interactive Search (CRIS) system was used to provide an anonymised, electronic mental health records database to search on structured data and free text fields on over 34,400 child and adolescent cases referred to SLaM services. Generalised Architecture for Text Engineering (GATE), a natural language processing architecture was applied to extract data from the free text (progress notes, correspondence, etc.) and structured fields [25]. 


\section{Study sample}

Cases were part of an open clinical cohort (entering and leaving the study at different time points) and included children aged 3-17 years with a diagnosis of ASD (International Classification of Diseases, 10th Revision (ICD10) F84.0, F84.1, F84.5, F84.9) [21] recorded between 1 January 2008 and 31 December 2013. Children were excluded if any past course of antipsychotic treatment was noted in the clinical record in the year prior to the observation period.

\section{Measurements}

\section{Outcome: antipsychotic use}

Antipsychotic use outcome data were extracted from free text fields held in the SLaM Case Register, the SLaM pharmacy dispensing database and structured medication fields in the electronic health record using validated GATE software extraction methods [26]. Antipsychotic use was measured during the observation period (01/01/2008-31/12/2013). The start date for antipsychotic use was recorded and categorised as a present/absent outcome.

\section{Exposure: psychiatric comorbidity and intellectual disability}

The main exposure was ICD-10 recorded comorbid psychiatric diagnoses, which were extracted from free text and structured fields. ICD-10 Axis one comorbid diagnoses were categorised into: psychotic (F1x.5, F20F29, F31, F32.3, F33.3), depressive disorders (F32), anxiety, stress and emotional (F40-41, F43-F48, F93), obsessive-compulsive (F42), hyperkinetic (F90), oppositional defiant and conduct (F91-F92) and tic (F95). Low frequency psychiatric diagnoses were collapsed into a single category labelled "Other". In addition, children were categorised according to presence of an ICD-10 Axis three diagnosis of intellectual disability (F70-F79).

Prescribing decisions were recorded contemporaneously, but there was often a short administrative lag before diagnostic reports appeared in the electronic medical record. These reports contained detailed clinical assessments conducted during the pre-medicated period. To permit inclusion of these longitudinally collected clinical data, but also ensure comorbidity exposures occurred prior to antipsychotic use, comorbid diagnoses were only coded as present if they were recorded before, or up to 30 days after, recording of antipsychotic medication.

\section{Covariates}

All covariates were extracted from the medical record during the initial CAMHS assessment period, and prior to antipsychotic use. Measures of challenging behaviours were taken from the SLaM risk assessment proforma. We chose assessment items with high face validity for challenging behaviours, as described by expert consensus in national guidance [14], including physical aggression against self (self-injury), violence and aggression to others or property (aggression), harm through loss of self care such as not drinking or eating (self-neglect), impulsive and dangerous acts (high-risk behaviours), and habitual behaviours related to intellectual disability such as rocking or skin picking that can cause injury (ID-related harm). Clinicians rated severity along a 4-point categorical scale: 'None', 'Low', 'Moderate', or 'High' (available on request). For ease of clinical interpretation and to ensure adequate numbers in each category, this scale was converted into a binary variable for each behaviour domain (Moderate or High rating categorised as High risk $=1$; None or Low ratings, low risk $=0$ ). Children's adaptive functioning was rated using Children's Global Assessment Scale (CGAS) [27], except for those children with significant ID, where the Developmental Disabilities CGAS was used in some cases [28]. Higher scores (range 0-100) are associated with better functioning.

Demographic and family covariates consisted of gender, ethnicity, history of parental mental illness, and clinicianrated levels of parental concern for their child's symptoms at their initial presentation to CAMHS, which were retrieved using CRIS from structured fields in the source dataset. Age at CAMHS assessment was calculated at the date of the first recorded diagnosis of autism spectrum disorder within the clinical record. UK Census data provided small area (average 400 households) level deprivation scores [29].

Emotional, hyperactive and conduct problem domains were assessed via the caregiver versions of the 25 -item Strengths and Difficulties Questionnaire which has sound psychometric properties in clinical samples [30]. These were available in the clinical record for a third of the sample $(n=1234,35 \%)$.

\section{Analysis}

To authenticate clinically recorded common comorbid conditions (depression and anxiety, hyperkinetic and conduct disorders), we used a sub-group of the cohort with completed SDQs $(n=1234)$. Independent sample $t$ tests were used to test for statistical differences in parental reported SDQ psychopathology subscale scores (emotional, 
hyperactivity, conduct problem subscales) for children with and without these common comorbid conditions.

Logistic regression was used to examine whether antipsychotic use was predicted by demographic characteristics, psychiatric comorbidities, intellectual disability, adaptive function, behaviours that challenge, parental characteristics and neighbourhood deprivation. Multivariable analysis was then conducted to examine the effect of each of these variables on antipsychotic use after adjusting all other individual and contextual covariates (listed in Table 1). The following sensitivity analyses were carried out: (1) using nonaggregated challenging behaviour categories (4 levels) as the binary variable may have introduced residual confounding; (2) excluding those who came from outside the local catchment (these individuals may have had substantial contact with non-SLaM services not represented in the CRIS source dataset); (3) selecting those with only one comorbid disorder and modelling the effect of a single comorbidity on antipsychotic use (without adjusting for the full set of covariates). All analyses were conducted using Stata version 12.

\section{Results}

Over the 6-year observation period, we identified 3482 children aged below 18 years (2686 male and 796 female) with a diagnosis of ASD. The mean (SD) exposure to child mental health services, defined as the time between the date of recorded ASD diagnosis and the end of the observation period or date of 18th Birthday (whichever sooner) was 968 (597) days. Three hundred and forty-eight children were prescribed antipsychotics, mainly risperidone (55\%, $n=191)$ and aripiprazole (32 \%, $n=112)$. All were receiving adjunctive psycho-social interventions. Table 1 shows the characteristics of the total sample and those prescribed antipsychotics. Nearly $75 \%$ of children prescribed antipsychotics were in the adolescent age range (age 13-18 years), representing a sixfold risk (OR 6.29, $95 \%$ CI 3.40-12.1) relative to early childhood (age 3-6 years).

In our authentication analyses, we found that ASD children diagnosed with comorbid emotional (depression and anxiety), hyperkinetic or conduct disorders had significantly higher SDQ subscales scores within their respective SDQ domains (emotional, hyperactive, conduct) compared with children without the respective comorbid diagnosis (see supplementary Table 1). ICD-10 recorded comorbid psychiatric diagnoses were present in $54 \%$ of the sample, a quarter diagnosed with a hyperkinetic disorder, and $20 \%$ diagnosed with intellectual disability. Table 2 provides further details of comorbid psychiatric diagnoses by antipsychotic use. Antipsychotics were prescribed to approximately half of children with ASD and psychotic disorder, and over a quarter of children diagnosed with obsessivecompulsive disorder, or tic disorders.

In the adjusted model, positive associations with antipsychotic use remained significant for age at the time of assessment, clinician-rated aggression, self-injurious behaviour, and high parental concern for their child's symptoms at initial presentation (see Table 3 ). In addition, adaptive function and the presence of caregiver substance misuse showed strong inverse associations with antipsychotic use. Associations with ethnicity, caregiver mental illness and neighbourhood deprivation were non-significant. Table 4 shows that a number of comorbid ICD-10 mental disorders, even after adjustment for all other covariates and comorbidities, remained significantly associated with antipsychotic use including hyperkinetic (OR 1.44, $95 \% \mathrm{CI}$ 1.01-2.06), psychotic (OR 5.71, 3.3-10.6), depressive (2.36, 1.37-4.09), obsessive-compulsive (2.31, 1.16-4.61) and tic disorders $(2.76,1.09-6.95)$. These associations remained when antipsychotic use was compared between ASD children with no-comorbidity to those who had the specific comorbidity alone, rather than multiple comorbidities (for example, only comorbid hyperkinetic disorder, see supplementary Table 2).

Specified sensitivity analyses that used non-aggregated behaviour categories produced little change to the overall pattern of results in the fully adjusted models, with the direction and magnitude of effect being consistent across the comorbidities. Similarly, removing the children resident outside the local catchment area from the sample ( $n=1170,33 \%)$ produced little change, except for oppositional defiant and conduct disorder, where the sensitivity analysis produced very imprecise estimates due to the low number of children within the diagnostic category.

\section{Discussion}

In the largest study to date using non-administrative, clinical mental health records in ASD, we found antipsychotic prescribing for children with ASD was strongly associated with comorbidity. Intellectual disability and psychiatric comorbidities, including hyperkinetic, depression, psychotic, obsessive-compulsive and tic disorders, were all associated with antipsychotic treatment, even after controlling for clinician-rated challenging behaviour symptoms at initial assessment. We also found increasing age, aggression, self-injurious behaviour, level of adaptive function, and parental concern were all significant predictors of antipsychotic use.

The observed association between antipsychotic use and age is consistent with previous ASD studies $[2,31]$. Over two-thirds of children treated with antipsychotics were adolescents. This highlights the need for more trials 
Table 1 Individual and contextual characteristics of 3482 children with autism spectrum disorders and antipsychotic use referred to local and specialist child and adolescent mental health services

\begin{tabular}{|c|c|c|c|c|}
\hline \multirow[t]{2}{*}{ Child age category } & \multicolumn{2}{|c|}{ At CAMHS assessment } & \multicolumn{2}{|c|}{ At antipsychotic use } \\
\hline & $n(\%)$ & Mean (SD) & $n(\%)$ & Mean (SD) \\
\hline Early (3-6 years) & $362(10.4 \%)$ & $4.9(0.77)$ & $3(0.9 \%)$ & $5.7(0.3)$ \\
\hline Mid (6-12 years) & $1664(47.8 \%)$ & $9.0(1.69)$ & $89(25.6 \%)$ & $9.6(1.4)$ \\
\hline \multirow[t]{2}{*}{ Late (13-17 years) } & $1456(41.8 \%)$ & $14.8(1.66)$ & $256(73.5 \%)$ & $15.1(1.6)$ \\
\hline & \multicolumn{2}{|c|}{ Total sample $n=3482$} & \multicolumn{2}{|c|}{ Sample receiving antipsychotics $n=348$} \\
\hline Male & $2686(77.1 \%)$ & & $249(71.6 \%)$ & \\
\hline Female & $796(22.9 \%)$ & & $99(28.4 \%)$ & \\
\hline \multicolumn{5}{|l|}{ Ethnicity } \\
\hline White British & $1683(48.3 \%)$ & & $208(59.8 \%)$ & \\
\hline White other & $170(4.9 \%)$ & & $11(3.2 \%)$ & \\
\hline East Asian & $68(2.0 \%)$ & & $9(2.6 \%)$ & \\
\hline British/Black African & $651(18.7 \%)$ & & $57(16.4 \%)$ & \\
\hline British/Black Caribbean & $130(3.7 \%)$ & & $5(1.4 \%)$ & \\
\hline Mixed Heritage & $386(11.1 \%)$ & & $37(10.6 \%)$ & \\
\hline South Asian & $84(2.4 \%)$ & & $11(3.2 \%)$ & \\
\hline Not stated & $310(8.9 \%)$ & & $10(2.8 \%)$ & \\
\hline \multicolumn{5}{|l|}{ Adaptive function } \\
\hline \multicolumn{5}{|c|}{ Children's Global Assessment Scale (CGAS) ${ }^{\mathrm{a}}$} \\
\hline 0-25 (most impaired) & $174(5.7 \%)$ & & $50(15.0 \%)$ & \\
\hline $25-50$ & $1346(43.8 \%)$ & & $219(65.6 \%)$ & \\
\hline $50-75$ & $1465(47.7 \%)$ & & $62(18.5 \%)$ & \\
\hline $75-100$ & $89(2.90 \%)$ & & $3(0.9 \%)$ & \\
\hline \multicolumn{5}{|c|}{ Challenging behaviours self injury ${ }^{b}$} \\
\hline Low & $2368(83.3 \%)$ & & $171(57.0 \%)$ & \\
\hline High & $474(16.7 \%)$ & & $129(43.0 \%)$ & \\
\hline \multicolumn{5}{|l|}{ ID-related harm ${ }^{\mathrm{c}}$} \\
\hline Low & $1600(59.3 \%)$ & & $142(48.6 \%)$ & \\
\hline High & $1097(40.7 \%)$ & & $150(51.4 \%)$ & \\
\hline \multicolumn{5}{|l|}{ Aggression $^{\mathrm{d}}$} \\
\hline Low & $1724(59.7 \%)$ & & $100(32.3 \%)$ & \\
\hline High & $1165(40.3 \%)$ & & $210(67.7 \%)$ & \\
\hline \multicolumn{5}{|l|}{ Self-neglect ${ }^{\mathrm{e}}$} \\
\hline Low & $2562(89.5 \%)$ & & $231(75.0 \%)$ & \\
\hline High & $300(10.5 \%)$ & & $77(25.0 \%)$ & \\
\hline \multicolumn{5}{|l|}{ High-risk behaviours $^{\mathrm{f}}$} \\
\hline Low & $2175(76.9 \%)$ & & $161(53.5 \%)$ & \\
\hline High & $654(23.1 \%)$ & & $140(46.5 \%)$ & \\
\hline \multicolumn{5}{|l|}{ Family characteristics } \\
\hline \multicolumn{5}{|l|}{ Caregiver mental illness ${ }^{\mathrm{g}}$} \\
\hline No & $2309(77.1 \%)$ & & $247(77.2 \%)$ & \\
\hline Yes & $685(22.9 \%)$ & & $73(22.8 \%)$ & \\
\hline \multicolumn{5}{|l|}{ Caregiver substance misuse ${ }^{\mathrm{g}}$} \\
\hline No & $2793(93.3 \%)$ & & $304(95.0 \%)$ & \\
\hline Yes & $201(6.7 \%)$ & & $16(5.0 \%)$ & \\
\hline \multicolumn{5}{|l|}{ Parental concern ${ }^{\mathrm{h}}$} \\
\hline Low & $876(30.1 \%)$ & & $33(10.6 \%)$ & \\
\hline High & $2033(69.9 \%)$ & & $278(89.4 \%)$ & \\
\hline
\end{tabular}


Table 1 continued

\begin{tabular}{llc}
\hline & Total sample $n=3482$ & Sample receiving antipsychotics $n=348$ \\
\hline $\begin{array}{l}\text { Neighbourhood characteristics }{ }^{\mathrm{i}} \\
\text { Level of deprivation (tertiles) }\end{array}$ & & \\
1st (least deprived) & $1064(32.8 \%)$ & $142(44.4 \%)$ \\
2nd & $1093(33.7 \%)$ & $95(29.7 \%)$ \\
3rd (most deprived) & $1089(33.6 \%)$ & $83(25.9 \%)$ \\
\hline
\end{tabular}

Missing cases $={ }^{\mathrm{a}} 408,{ }^{\mathrm{b}} 640,{ }^{\mathrm{c}} 785,{ }^{\mathrm{d}} 593,{ }^{\mathrm{e}} 620,{ }^{\mathrm{f}} 653,{ }^{\mathrm{g}} 488,{ }^{\mathrm{h}} 573,{ }_{2}^{\mathrm{i}} 26$

that include this age group but also suggests that treatment acceptability, and hence trial recruitment, will be more feasible than in younger children. Social factors also appeared to play a role; clinicians who perceived greater parental concern for children's presenting symptoms were more likely to prescribe antipsychotic treatment. We are not aware of any prior studies that measure parental influences on antipsychotic use in ASD; however, our finding is consistent with previous studies that show a positive association between parental strain and medication treatment for childhood disruptive disorders [32]. Consistent with several other investigations in clinical samples [33, 34], our unadjusted analyses suggest that there may be discrepancies between ethnic groups regarding prescribing antipsychotic medication to children. However, in keeping with a more recent study on psychotropic treatment in children, we found that after adjustment for markers of clinical severity, ethnicity was no longer significantly associated with antipsychotic use [35].

Using a historical cohort design in a clinical sample of children with ASD, this is the first longitudinal study of challenging behaviours and psychiatric comorbidity profiles as predictors of antipsychotic use. Our results suggest that clinicians are using antipsychotics where they are known to be efficacious [7]; to target aggression and self-injurious behaviours. Many studies so far have been hindered by parental report of comorbidities and medication use, retrospective or cross-sectional design, or the confounding effect of unmeasured psychiatric symptoms and disorder severity not being accounted for $[2,3,36]$. In addressing these limitations we found that, unlike a number of US studies, antipsychotics were not significantly associated with comorbid anxiety, emotional and stress disorders $[2,3,36]$.

This study has a number of strengths: we used longitudinally collected clinician recorded data in an unselected population of children and adolescents with ASD referred to CAMHS to study off-label antipsychotic use. This avoids the non-response or recall bias issues that may arise in surveys of parents. Our sample included the entire psychiatric population of four south London boroughs for school
Table 2 Prevalence of comorbid psychiatric disorder and antipsychotic treatment in 3482 children with autism spectrum disorders

\begin{tabular}{|c|c|c|}
\hline & Total sample $n=3482$ & $\begin{array}{l}\text { Sample receiving antipsychotics } \\
n=348\end{array}$ \\
\hline \multicolumn{3}{|c|}{ Any comorbid disorder } \\
\hline- & $1585(45.5 \%)$ & $63(18.1 \%)$ \\
\hline+ & $1897(54.5 \%)$ & $285(81.9 \%)$ \\
\hline \multicolumn{3}{|c|}{ Hyperkinetic } \\
\hline- & $2620(75.2 \%)$ & $227(65.2 \%)$ \\
\hline+ & $862(24.8 \%)$ & $121(34.8 \%)$ \\
\hline \multicolumn{3}{|c|}{ Oppositional and conduct } \\
\hline- & $3226(92.7 \%)$ & $297(85.3 \%)$ \\
\hline+ & $256(7.3 \%)$ & $51(14.7 \%)$ \\
\hline \multicolumn{3}{|c|}{ Depression } \\
\hline- & $3328(95.6 \%)$ & $312(89.7 \%)$ \\
\hline+ & $154(4.4 \%)$ & $36(10.3 \%)$ \\
\hline \multicolumn{3}{|c|}{ Anxiety, emotional and stress } \\
\hline- & $3203(92.0 \%)$ & $303(87.1 \%)$ \\
\hline+ & $279(8.0 \%)$ & $45(12.9 \%)$ \\
\hline \multicolumn{3}{|c|}{ Obsessive-compulsive } \\
\hline- & $3385(97.2 \%)$ & $322(92.5 \%)$ \\
\hline+ & $97(2.8 \%)$ & $26(7.5 \%)$ \\
\hline \multicolumn{3}{|c|}{ Tic } \\
\hline- & $3431(98.5 \%)$ & $335(96.3 \%)$ \\
\hline+ & $51(1.5 \%)$ & $13(3.7 \%)$ \\
\hline \multicolumn{3}{|c|}{ Psychosis } \\
\hline- & $3366(96.8 \%)$ & $294(84.5 \%)$ \\
\hline+ & $116(3.3 \%)$ & $54(15.5 \%)$ \\
\hline \multicolumn{3}{|c|}{ Intellectual disability } \\
\hline- & $2826(81.2 \%)$ & $234(67.2 \%)$ \\
\hline+ & $656(18.8 \%)$ & $114(32.8 \%)$ \\
\hline \multicolumn{3}{|c|}{ Other** } \\
\hline- & $3353(96.3 \%)$ & $330(94.8 \%)$ \\
\hline+ & $129(3.7 \%)$ & $18(5.2 \%)$ \\
\hline
\end{tabular}

$(-)$ comorbidity absent $(+)$ comorbidity present

**Remaining, rarely occurring diagnoses, were collapsed into a single category labelled Other (includes ICD-10 F50 eating disorders, F04-09 organic disorders, F1x.1-4 substance misuse, F94.1-2 attachment disorders) 
Table 3 Multivariable model of antipsychotic use in children with ASD by socio-demographic characteristics and other covariates

\begin{tabular}{|c|c|c|c|c|}
\hline & OR $(95 \%$ CI $)(n=3482)$ & $P$ & $\mathrm{aOR}(95 \% \mathrm{CI})^{\mathrm{a}}$ & $P$ \\
\hline Female sex (vs male) & $1.39(1.09-1.79)$ & 0.009 & $1.02(0.71-1.46)$ & 0.89 \\
\hline Age at CAMHS assessment & $1.18(1.15-1.23)$ & $<0.0001$ & $1.11(1.05-1.16)$ & $<0.001$ \\
\hline \multicolumn{5}{|l|}{ Ethnicity } \\
\hline White British & Reference & & & \\
\hline White other & $0.49(0.26-0.92)$ & 0.026 & $0.62(0.24-1.55)$ & 0.31 \\
\hline East Asian & $1.08(0.52-2.21)$ & 0.83 & $0.91(0.35-2.31)$ & 0.84 \\
\hline British/Black African & $0.68(0.50-0.92)$ & 0.014 & $1.14(0.73-1.78)$ & 0.55 \\
\hline British/Black Caribbean & $0.28(0.11-0.70)$ & 0.006 & $0.56(0.21-1.54)$ & 0.26 \\
\hline Mixed Heritage & $0.75(0.52-1.09)$ & 0.13 & $0.78(0.46-1.32)$ & 0.36 \\
\hline South Asian & $1.06(0.56-2.05)$ & 0.84 & $1.26(0.47-3.32)$ & 0.70 \\
\hline Not stated & $0.24(0.12-0.45)$ & $<0.001$ & $0.27(0.09-0.80)$ & 0.02 \\
\hline \multicolumn{5}{|l|}{ Adaptive function } \\
\hline Children's Global Assessment Scale (CGAS) & $0.95(0.94-0.95)$ & $<0.0001$ & $0.96(0.95-0.97)$ & $<0.0001$ \\
\hline \multicolumn{5}{|l|}{ Challenging behaviours } \\
\hline Self-injury & $4.80(3.72-6.20)$ & $<0.0001$ & $1.85(1.30-2.63)$ & $<0.0001$ \\
\hline ID-related harm & $1.63(1.27-2.07)$ & $<0.0001$ & $0.72(0.49-1.06)$ & 0.10 \\
\hline Aggression & $3.57(2.77-4.59)$ & $<0.0001$ & $2.14(1.50-2.06)$ & $<0.0001$ \\
\hline Self-neglect & $3.48(2.61-4.67)$ & $<0.0001$ & $1.20(0.78-1.80)$ & 0.35 \\
\hline High-risk behaviours & $3.40(2.66-4.35)$ & $<0.0001$ & $1.22(0.86-1.73)$ & 0.27 \\
\hline \multicolumn{5}{|l|}{ Family characteristics } \\
\hline Caregiver mental illness & $1.0(0.75-1.31)$ & 0.98 & $0.87(0.60-1.26)$ & 0.47 \\
\hline Caregiver substance misuse & $0.71(0.42-1.20)$ & 0.20 & $0.57(0.30-1.08)$ & 0.09 \\
\hline High parental concern & $4.05(2.79-5.85)$ & $<0.0001$ & $2.02(1.27-3.22)$ & 0.003 \\
\hline \multicolumn{5}{|l|}{ Neighbourhood } \\
\hline \multicolumn{5}{|l|}{ Level of deprivation (tertiles) } \\
\hline 1st (least deprived) & Reference & & & \\
\hline 2 nd & $0.62(0.47-0.81)$ & 0.001 & $0.82(0.56-1.19)$ & 0.31 \\
\hline 3rd (most deprived) & $0.54(0.40-0.71)$ & $<0.0001$ & $0.91(0.62-1.35)$ & 0.65 \\
\hline
\end{tabular}

OR odds ratio, $C I$ confidence intervals

a aOR, adjusted Odds Ratio, fully adjusting for all other covariates within the table, i.e. socio-demographic (age at CAMHS assessment, gender and ethnicity) and parental and neighbourhood characteristics, challenging behaviours, adaptive function and co-existing ICD-10 mental and behavioural disorder groupings: hyperkinetic (F90), depressive disorders (F32), psychosis (F1x.5, F20-F29, F31, F32.3, F33.3) oppositional and conduct (F91-F92), anxiety, stress and emotional (F40-41, F43-F48, F93), obsessive-compulsive (OCD, F42), tic (F95), intellectual disability (ID, F70-F79) and other psychiatric diagnosis

age children (4-18 years) with suspected or previously confirmed ASD and displaying emotional or behavioural difficulties, in addition to children from other areas of the UK referred to National and Specialist services. However, because we studied a cohort enriched by national referrals, psychiatric comorbidity and antipsychotic treatment, prevalence should not be taken as representative rates of the children with ASD in the general population.

Our study has limitations. First, an ASD diagnosis may 'overshadow' other psychiatric diagnoses and reduce the likelihood of clinicians recording additional psychiatric diagnoses. For example, ICD-10 criteria preclude the diagnosis of hyperkinetic disorder being given once ASD is established, which may lead to an underestimate of the association between hyperkinetic comorbidity and antipsychotic use. That said, many clinicians override this instruction based on recent evidence from clinical and treatment studies. Second, the type of assessment and treatments offered to families may vary by clinician. In our analysis, we lacked detailed information about the assessing and prescribing clinician and could not account for variation in practice. Third, we did not include physical comorbidities (e.g. epilepsy, obesity), other pharmacological treatments or duration of psycho-social interventions which may act as potential confounders to antipsychotic use. Fourth, we did not apply a research scale to measure challenging behaviours [37]. Instead, we used assessment items commonly mandated for use in clinical mental health services [38], 
Table 4 Multivariable model of antipsychotic use in a cohort of children with ASD by psychiatric comorbidity

\begin{tabular}{lclll}
\hline & $\mathrm{OR}(95 \% \mathrm{CI})(n=3482)$ & $P$ & $\mathrm{aOR}^{\mathrm{a}}(95 \% \mathrm{CI})$ & $P$ \\
\hline Any comorbid disorder & $4.27(3.22-5.66)$ & $<0.0001$ & - & - \\
Hyperkinetic & $1.73(1.36-2.18)$ & $<0.0001$ & $1.44(1.01-2.06)$ & 0.042 \\
Oppositional and conduct & $2.47(1.77-3.43)$ & $<0.0001$ & $1.55(0.96-2.51)$ & 0.073 \\
Depression & $2.95(1.99-4.36)$ & $<0.0001$ & $2.36(1.37-4.09)$ & 0.002 \\
Anxiety, emotional and stress & $1.84(1.31-2.59)$ & $<0.0001$ & $1.20(0.72-1.98)$ & 0.484 \\
Obsessive-compulsive & $3.48(2.19-5.53)$ & $<0.0001$ & $2.31(1.16-4.61)$ & 0.017 \\
Tic & $3.16(1.67-5.99)$ & $<0.0001$ & $2.76(1.09-6.95)$ & 0.032 \\
Psychosis & $9.1(6.19-13.4)$ & $<0.0001$ & $5.71(3.28-10.6)$ & $<0.0001$ \\
Intellectual disability & $2.33(1.82-2.97)$ & $<0.0001$ & $1.68(1.11-2.53)$ & 0.015 \\
Other $^{\mathrm{b}}$ & $1.49(0.89-2.48)$ & 0.13 & $1.62(0.83-3.16)$ & 0.157 \\
\hline
\end{tabular}

a aOR, adjusted Odds Ratio, adjusting for socio-demographic and parental and neighbourhood characteristics, challenging behaviours, adaptive function and co-existing ICD-10 Mental and behavioural disorder groupings: hyperkinetic (F90), depressive disorders (F32), psychosis (F1x.5, F20-F29, F31, F32.3, F33.3), oppositional and conduct (F91-F92), anxiety, stress and emotional (F40-41, F43-F48, F93), obsessivecompulsive (OCD, F42), tic (F95), intellectual disability (ID, F70-F79) and other psychiatric diagnosis

b Remaining, rarely occurring diagnoses, were collapsed into a single category labelled Other (includes ICD-10 F50 eating disorders, F04-09 organic disorders, F1x.1-4 substance misuse, F94.1-2 attachment disorders) which could likely aid study replication in other UK settings. Fifth, we coded comorbid disorders preceding and up to 30 days post antipsychotic use, which prevented the exclusion of pre-medication diagnostic reports. Theoretically, this could introduce an observer bias, as the intensity of observation by the CAMHS service post antipsychotic treatment may increase a child's risk of having a clinically recorded comorbid disorder. However, iatrogenic comorbid psychiatric conditions are very unlikely to develop or be recorded within this short timeframe. Last, due to limitations in the free text coding and extraction process, we cannot exclude residual confounding as an influence on our findings, especially within the broad diagnostic categories of psychotic disorder or intellectual disability. We were unable to accurately categorise the degree of intellectual disability, nor characterise the severity or duration of psychotic disorder from the electronic health records. However, we did address potential confounding due to severity of psychotic disorders and intellectual disability to some extent by the inclusion of Children's Global Assessment Score [27] as a covariate in the final multivariable models. Residual confounding may remain nonetheless.

Our findings reflect the complexity of assessing and treating comorbid psychiatric disorders in ASD. For example, ASD and psychotic disorders pose a common diagnostic challenge to clinicians given their overlapping characteristics and high potential for co-occurrence [39, 40]. We found only $47 \%$ of children with ASD and psychosis received antipsychotics. This low treatment rate may be due to diagnostic uncertainty. Children with ASD may be more likely to have their diagnosis of psychosis withdrawn after further clinical assessment, and before the initiation of antipsychotic treatment. A second reason may relate to clinicians, children and their families deciding that some psychotic symptoms in ASD do not warrant antipsychotic treatment. Evidence that may dissuade those from starting antipsychotic treatment includes findings from longitudinal studies, which show fluctuating psychotic symptoms in children with features of autism can have a relatively benign course [41, 42].

Our findings provide a detailed account of current antipsychotic prescribing practices in a clinical population of children with ASD, which show that aggression and selfinjurious behaviours are significantly associated with antipsychotic use. Irritability may be an underlying treatment target driving the association between these behaviours and antipsychotic treatment. It may also underlie the associations we found between antipsychotic use and hyperkinetic, depressive and obsessive-compulsive disorders. Alternatively, disorder specific symptoms may be targeted. For example, trial data have shown that risperidone and aripiprazole both significantly reduce hyperactivity and obsessional compulsive symptoms in ASD [37, 43]. In addition, the study findings highlight the need for further research in childhood ASD to determine which psychotic phenomena warrant antipsychotic treatment. This would help clinicians reduce the harms associated with both antipsychotic underuse (i.e. prolonging the duration of untreated psychosis) and over-use. Future research might valuably include children without ASD as comparison groups and employ more intricate text extraction methodologies to assess symptom-specific severity and impairment. This would reduce the residual confounding effects that may occur when using broad diagnostic categories, and determine whether 
comorbid psychiatric diagnoses in clinical practice are approached differently in children with ASD.

Our findings highlight a mismatch between current clinical trials and the evidence needed to support clinical practice in ASD. Antipsychotic use was much greater in adolescents and for those with comorbid diagnoses. However, most published trials exclude children with comorbidity and rarely recruit adolescents $[7,8,11]$. Importantly, we show social factors play a significant part in antipsychotic use. This provides an impetus to examine the association of antipsychotic treatment against contextual, as well as clinical factors. Controversy between the potential harm of both over- and under-use of antipsychotics in children with ASD continues, and underlies considerable public concern [15]. Large-scale cohort studies in real world settings, such as ours, eventually leading to pragmatic trials using electronic patient records, will help this debate become better informed.

Acknowledgments This research was supported by the Biomedical Research Nucleus data management and informatics facility at South London and Maudsley NHS Foundation Trust, which is funded by the National Institute for Health Research (NIHR) Biomedical Research Centre for Mental Health at South London and Maudsley NHS Foundation Trust and King's College London and a joint infrastructure Grant from Guy's and St. Thomas' Charity and the Maudsley Charity. Johnny Downs is supported by a UK Medical Research Council (MRC) Clinical Research Training Fellowship (MR/L017105/1). Richard Hayes is supported by a Medical Research Council (MRC) Population Health Scientist Fellowship.

\section{Compliance with ethical standards}

Ethics statement CRIS was approved as an anonymised data resource for secondary analysis by Oxfordshire Research Ethics Committee $\mathrm{C}(08 / \mathrm{H} 0606 / 71+5)$, and have therefore been performed in accordance with the ethical standards laid down in the 1964 Declaration of Helsinki and its later amendments. All projects using CRIS are reviewed and approved by a dedicated patient-led oversight committee.

Declaration of interest $\mathrm{RH}, \mathrm{RJ}, \mathrm{HS}$, and RS have received research funding from Roche, Pfizer, J\&J and Lundbeck. Other authors have no conflict of interest.

Open Access This article is distributed under the terms of the Creative Commons Attribution 4.0 International License (http://creativecommons.org/licenses/by/4.0/), which permits unrestricted use, distribution, and reproduction in any medium, provided you give appropriate credit to the original author(s) and the source, provide a link to the Creative Commons license, and indicate if changes were made.

\section{References}

1. Hsia Y, Wong AYS, Murphy DGM et al (2013) Psychopharmacological prescriptions for people with autism spectrum disorder (ASD): a multinational study. Psychopharmacology 231:999-1009
2. Coury DL, Anagnostou E, Manning-Courtney P et al (2012) Use of psychotropic medication in children and adolescents with autism spectrum disorders. Pediatrics 130:S69-S76

3. Mandell DS, Morales KH, Marcus SC et al (2008) Psychotropic medication use among medicaid-enrolled children with autism spectrum disorders. Pediatrics 121:e441-e448

4. Murray ML, Hsia Y, Glaser K et al (2013) Pharmacological treatments prescribed to people with autism spectrum disorder (ASD) in primary health care. Psychopharmacology 231:1011-1021

5. Bachmann CJ, Manthey T, Kamp-Becker I et al (2013) Psychopharmacological treatment in children and adolescents with autism spectrum disorders in Germany. Res Dev Disabil 34:2551-2563

6. Rani F, Murray ML, Byrne PJ, Wong IC (2008) Epidemiologic features of antipsychotic prescribing to children and adolescents in primary care in the United Kingdom. Pediatrics 121:1002-1009

7. McPheeters ML, Warren Z, Sathe N et al (2011) A systematic review of medical treatments for children with autism spectrum disorders. Pediatrics 127:e1312-e1321

8. American Academy of Child and Adolescent Psychiatry Policy Statement on Comorbidity Treatment in Autism Spectrum Disorders and Intellectual Disabilities. http://www.aacap.org/ aacap/Policy_Statements/2013/Comorbidity_Treatment_in_ Autism_Spectrum_Disorders_and_Intellectual_Disabilities.aspx. Accessed 11 Jul 2015

9. Stringaris A (2011) Irritability in children and adolescents: a challenge for DSM-5. Eur Child Adolesc Psychiatry 20:61-66

10. National Institute for Health and Care Excellence (2014) Antisocial behaviour and conduct disorders in children and young people. QS59. National Institute for Health and Care Excellence, London

11. Dove D, Warren Z, McPheeters ML et al (2012) Medications for adolescents and young adults with autism spectrum disorders: a systematic review. Pediatrics 130:717-726

12. Lin J-D (2014) Medical care burden of children with autism spectrum disorders. Rev J Autism Dev Disord 1:242-247

13. Almandil NB, Liu Y, Murray ML et al (2013) Weight gain and other metabolic adverse effects associated with atypical antipsychotic treatment of children and adolescents: a systematic review and meta-analysis. Paediatr Drugs 15:139-150

14. National Institute for Health and Care Excellence (2013) Autism: the management and support of children and young people on the autism spectrum. CG 170. National Institute for Health and Care Excellence, London

15. Glover G, Bernard S, Branford D et al (2014) Use of medication for challenging behaviour in people with intellectual disability. Br J Psychiatry 205:6-7

16. Simonoff E, Pickles A, Charman T et al (2008) Psychiatric disorders in children with autism spectrum disorders: prevalence, comorbidity, and associated factors in a population-derived sample. J Am Acad Child Adolesc Psychiatry 47:921-929

17. Every-Palmer S, Howick J (2014) How evidence-based medicine is failing due to biased trials and selective publication. J Eval Clin Pract 20:908-914

18. US Department of Health and Human Services Aripiprazole: Pediatric Labeling Information. http://www.accessdata.fda.gov/ scripts/cder/drugsatfda/index.cfm?fuseaction=Search.Overview $\&$ DrugName $=$ ABILIFY8. Accessed 10 Jul 2015

19. US Department of Health and Human Services Risperidone : Pediatric Labeling Information. http://www.accessdata.fda.gov/ scripts/cder/drugsatfda/index.cfm?fuseaction=Search.Overview $\&$ DrugName $=$ RISPERIDONE. Accessed 10 Jul 2015

20. Taylor E (2013) Pediatric psychopharmacology: too much and too little. World Psychiatry 12:124-125 
21. World Health Organisation (2008) Multiaxial classification child and adolescent psychiatric disorders: the ICD-10 classification of mental and behavioural disorders in children and adolescents. WHO, Cambridge

22. Lord C, Rutter M, Goode S et al (1989) Austism diagnostic observation schedule: a standardized observation of communicative and social behavior. J Autism Dev Disord 19:185-212

23. Baird G, Simonoff E, Pickles A et al (2006) Prevalence of disorders of the autism spectrum in a population cohort of children in South Thames: the special needs and autism project (SNAP). Lancet 368:210-215

24. Goodman R (1997) The strengths and difficulties questionnaire: a research note. J Child Psychol Psychiatry 38:581-586

25. Wu C-Y, Chang C-K, Robson D et al (2013) Evaluation of smoking status identification using electronic health records and opentext information in a large mental health case register. PLoS ONE 8:e74262

26. Hayes RD, Downs J, Chang C-K et al (2015) The effect of clozapine on premature mortality: an assessment of clinical monitoring and other potential confounders. Schizophr Bull 41:644-655

27. Shaffer D, Gould MS, Brasic J et al (1983) A children's global assessment scale (CGAS). Arch Gen Psychiatry 40:1228-1231

28. Wagner A, Lecavalier L, Arnold LE, Aman MG (2007) Developmental disabilities modification of the children's global assessment scale. Biol Psychiatry 61:504-511

29. Department for Communities and Local Government English indices of deprivation 2010: technical report

30. Goodman R (2001) Psychometric properties of the strengths and difficulties questionnaire. J Am Acad Child Adolesc Psychiatry 40:1337-1345

31. Rubin DM, Feudtner C, Localio R, Mandell DS (2009) State variation in psychotropic medication use by foster care children with autism spectrum disorder. Pediatrics 124:e305-e312

32. Garland AF, Brookman-Frazee L, Gray E (2013) The role of parent characteristics in community-based medication treatment for children with disruptive behavior problems. Community Ment Health J 49:507-514

33. Leslie LK, Weckerly J, Landsverk J, Hough RL (2003) Racial/ ethnic differences in the use of psychotropic medication in high-risk children and adolescents. J Am Acad Child Adolesc Psychiatry 42:1433-1442

34. Zuvekas SH, Vitiello B, Norquist GS (2006) Recent trends in stimulant medication use among U.S. children. Am J Psychiatry 163:579-585

35. Drilea SK, Jowers K, Lichtenstein C et al (2013) Psychotropic medication use and clinical outcomes among children and adolescents receiving system of care services. J Child Adolesc Psychopharmacol 23:36-43

36. Frazier TW, Shattuck PT, Narendorf SC et al (2011) Prevalence and correlates of psychotropic medication use in adolescents with an autism spectrum disorder with and without caregiverreported attention-deficit/hyperactivity disorder. J Child Adolesc Psychopharmacol 21:571-579

37. Aman MG, Kasper W, Manos G (2010) Line-item analysis of the Aberrant Behavior Checklist: results from two studies of aripiprazole in the treatment of irritability associated with autistic disorder. J Child Adolesc Psychopharmacol 20:415-422

38. Hawley CJ, Littlechild B, Sivakumaran T (2006) Structure and content of risk assessment proformas in mental healthcare. J Ment Health 15:437-448

39. Waris P, Lindberg N, Kettunen K, Tani P (2013) The relationship between Asperger's syndrome and schizophrenia in adolescence. Eur Child Adolesc Psychiatry 22:217-223

40. Kyriakopoulos M, Stringaris A, Manolesou S et al (2015) Determination of psychosis-related clinical profiles in children with autism spectrum disorders using latent class analysis. Eur Child Adolesc Psychiatry 24:301-307

41. Kumra S, Jacobsen LK, Lenane M et al (1998) "Multidimensionally impaired disorder": is it a variant of very early-onset schizophrenia? J Am Acad Child Adolesc Psychiatry 37:91-99

42. Stayer C, Sporn A, Gogtay N et al (2005) Multidimensionally impaired: the good news. J Child Adolesc Psychopharmacol 15:510-519

43. McDougle CJ, Scahill L, Aman MG et al (2005) Risperidone for the core symptom domains of autism: results from the study by the autism network of the research units on pediatric psychopharmacology. Am J Psychiatry 162:1142-1148 